\title{
A Brief Tutorial on Acceptance and Commitment Therapy as Seen Through the Lens of Derived Stimulus Relations
}

\author{
Ciara McEnteggart ${ }^{1}$ (D)
}

Published online: 30 April 2018

(C) Association for Behavior Analysis International 2018

Acceptance and commitment therapy (ACT; Hayes, Strosahl, \& Wilson, 1999) has been identified as a modern behavior therapy and has a substantive body of supporting evidence that includes over 200 randomized controlled trials. ACT has been linked directly to relational frame theory (RFT; Hayes, Barnes-Holmes, \& Roche, 2001) - a behavior-analytic account of human language and cognition that relies on the concept of derived stimulus relations. RFT also has a growing body of empirical evidence (Barnes-Holmes, Finn, McEnteggart, \& Barnes-Holmes, in press; O’Connor, Farrell, Munnelly, \& McHugh, 2017). The basic idea behind both RFT and ACT is that the evolution of human language, conceptualized as derived relational responding, creates a type of psychological suffering that is largely unique to humans. RFT constitutes the basic theory behind this assertion, and ACT, as a clinical intervention, is designed to deal with the psychological problems that human language creates for our species (Törneke, 2010; Villatte, Villatte, \& Hayes, 2015).

This article provides a tutorial on the links between ACT and derived stimulus relations, particularly through the lens of RFT. Although RFT is sometimes seen as providing the basic behavioral science underpinning ACT, it is perhaps more accurate to say that they have co-evolved. Thus, RFT did not give rise directly to ACT, and ACT did not backward engineer RFT, but the two are interrelated. This tutorial thus aims to articulate the clear links between the theory and the application (where they exist) and how RFT suggests functional-analytic ways in which clinically relevant behavior can be understood and manipulated. This article will first describe the conceptual

This article was prepared with the support of an Odysseus Group I grant awarded to Prof. Dermot BarnesHolmes by the Flanders Science Foundation (FWO). The author would like to thank Dermot Barnes-Holmes and Yvonne Barnes-Holmes for their valuable comments on the article.

Ciara McEnteggart

ciara.mcenteggart@ugent.be

1 Department of Experimental Clinical and Health Psychology, Ghent University, Henri Dunantlaan 2, 9000 Ghent, Belgium 
background of ACT, followed by an overview of four key areas in which RFT is clearly linked to ACT and potentially other forms of psychotherapy. These areas include (a) transformations of function, (b) rule-governed behavior, (c) relating relations and analogy, and (d) the verbal self. The article will then briefly outline a recent conceptual development within RFT and how this may also connect with ACT.

Before continuing, it is important to emphasize, that ACT and the study of derived relations (in terms of RFT) did not emerge in a linear sequence (e.g., first RFT, then ACT) but rather co-evolved. Early in the history of ACT (and RFT), it could be argued that the connection between the two was relatively clear, at least in some key areas. However, ACT quickly grew in popularity, and the need for a model for training clinicians and a model for presenting ACT to verbal communities not well versed in behavior analysis (let alone derived relations research and RFT) quickly became apparent. This led to the development of the psychological flexibility model known as the hexaflex (e.g., Hayes, Luoma, Bond, Masuda, \& Lillis, 2006). Although the development of this model clearly had some benefits, it also made it increasingly difficult to see the connections between ACT and RFT (see Barnes-Holmes, Hussey, McEnteggart, Barnes-Holmes, \& Foody, 2016, for a detailed discussion). The current article suggests, however, that connections remain and that indeed it may be possible to develop and refine them within the context of conceptual developments in RFT that may emerge over the coming years. The potential advantages of maintaining those links between the basic science and application remain to be determined, but it seems important to do so with the increasing emphasis that is being placed on basic processes in evaluating the impact and value of psychotherapy generally.

\section{ACT: Conceptual Background and Its Early Connection to the Study of Derived Stimulus Relations}

Arguably, one of the first steps toward a contextual approach to psychological therapy was made by applied behavior analysts who used consequential and discriminative stimulus control to change socially important behaviors (Baer, Wolf, \& Risley, 1968). Specifically, functional assessments were used to identify the contextual variables that exerted stimulus and consequential control over specific target behaviors. These contextual variables could then be used to bring about desired behavior change. When these variables were approached in less contextually sensitive and sophisticated ways, the technology transformed into what has sometimes been referred to as behavior modification, in which the role of consequences to change behavior overshadowed functional assessment (Hayes, Rincover, \& Solnick, 1980; Zettle, 2016). The importance of functional assessment re-emerged, however, when researchers returned to a more contextual approach to challenging behaviors, particularly within the domain of developmental disabilities (Carr, 1977, 1994; Hanley, Iwata, \& McCord, 2003; Iwata, Dorsey, Slifer, Bauman, \& Richman, 1982; Luiselli \& Cameron, 1998; O’Neill, Horner, Albin, Story, \& Sprague, 1990). Despite this re-emergence and its success with developmental disabilities, it has been argued that applied behavior analysis has remained limited in its ability to deal with the complexities of clinical phenomena among verbally sophisticated adults (Dymond \& Alonso-Álvarez, 2010; Dymond, O’Hora, Whelan, \& O’Donovan, 2006; Friman, 2006; Hayes, 2001). 
Perhaps one could argue that the relatively narrow focus of applied behavior analysis in the clinical domain facilitated the emergence of cognitive behavioral therapy (CBT; Beck, 1976) as a way of dealing with verbally sophisticated individuals. As part of the so-called cognitive revolution, CBT was very much focused on mediating cognitive or mental events that were used to explain "pathological" behavior. For example, CBT used the concept of cognitive control to describe how cognitions (i.e., thoughts or schemata) control actions and thus cause emotional and behavioral problems (Zettle, 1990). This view highlighted the role of human language and cognition in human psychological suffering, but with a very strong focus on mental representations and processes to guide psychotherapy. As such, an emphasis on the environment, or an individual's history of experience within that environment, seemed to become less important, at least in terms of generating explanatory concepts. Put simply, from the cognitive perspective, individuals who suffered psychologically were deemed to have abnormal cognitions, dysfunctional schemata, or inappropriate appraisals. The "problem" thus lay within the mind of the individual and not in the interaction of the individual with his or her environment - or, to put it bluntly, minds were seen to be "sick" or "dysfunctional" rather than the environments in which individuals lived being seen as problematic.

ACT could be viewed, therefore, as an attempt within the behavior-analytic tradition to develop a therapy that was contextually and behaviorally focused but that also recognized the pivotal role that human language and cognition play in psychological suffering. ${ }^{1}$ An early behavior-analytic attempt to grapple with language and cognition used a concept first proposed in the mid-1960s: that of instructional control or rulegoverned behavior (Skinner, 1966). Specifically, Skinner (1969) defined rules as contingency-specifying stimuli: "We tend to follow rules because previous behavior in response to similar verbal stimuli has been reinforced" (p. 148). And indeed, from the beginning, ACT emphasized excessive rule following as a source of human suffering. One method to deal with this excessive rule following was known as cognitive distancing, which was defined as the ability to observe or "step back" from thoughts and feelings (Zettle \& Hayes, 1983). That is, from a behavior-analytic perspective, clients were taught to observe or simply notice problematic rules that had been used to guide behavior and to engage more directly with the contingencies of reinforcement in the natural environment (Zettle, 2016). Indeed, during the first decade of the inception of ACT, the therapy was referred to as comprehensive distancing.

Early ACT drew heavily on the concept of rule-governed behavior at a time when this type of behavior was attracting considerable attention in the basic science of behavior analysis (see Hayes, 1989, for an extensive review). Despite the body of literature that had emerged up until this point, no precise functional-analytic definition of instructional control or rule-governed behavior had been offered. More precisely,

\footnotetext{
${ }^{1}$ ACT was developed initially for largely clinical purposes, but given its roots in a functional analysis of human language and cognition, it has been expanded to cover a wide range of applied contexts. For example, ACT has been used in organizational psychology (e.g., Bond, Hayes, \& Barnes-Holmes, 2006), the treatment of epilepsy (e.g., Lundgren, Dahl, \& Hayes, 2008), palliative care (e.g., Feros, Lane, Ciarrochi, \& Blackledge, 2013), stigmatization and prejudice (e.g., Lillis \& Hayes, 2007), and parenting children with intellectual disabilities (e.g., Burke et al., 2014).
} 
Skinner had argued that rules specify reinforcement contingencies, but the functional definition of specification itself had remained ill defined. A functional-analytic definition of specification, however, was provided in part by Sidman's seminal body of work on stimulus equivalence (e.g., 1971). In brief, equivalence relations were defined by the emergence of matching behaviors that could not be explained by direct reinforcement contingencies or other well-established behavioral principles. For example, a child who was taught to match a spoken word to an object and a spoken word to a printed word was able to spontaneously match the printed word to the object without any further reinforcement (see Sidman, 1994). Sidman proposed that the formation of such equivalence relations may provide a behavior-analytic interpretation of semantic meaning in human language. In so doing, it could be argued that Sidman provided the muchneeded definition of specification that was missing in the early behavioral literature on rule-governed behavior.

Whereas Sidman's work on equivalence relations focused on what may be considered the most basic type of symbolic relation, Hayes and colleagues subsequently argued that equivalence responding may be interpreted as a type of generalized operant behavior. Moreover, many such operants could be identified within natural human language, and these were referred to as relational frames. Thus, for example, children not only learned to relate arbitrary stimuli as participating in equivalence relations but also acquired other relational operants, such as coordination, opposition, distinction, comparison, spatial frames, temporal frames, deictic relations, and hierarchical relations (see Hughes \& Barnes-Holmes, 2016, for a recent review). The concept of the relational frame as a generalized relational operant has acquired increasing support and seems to provide a functional-analytic way of defining and studying rule-governed behavior.

According to RFT, a rule or instruction is typically composed of a network of frames of equivalence and temporal relations. Broadly speaking, the equivalence relations serve to specify the events in the relevant contingency, with the temporal relations coordinating the rule with the contingency itself. Thus, for example, the simple rule "When the green light comes on, then go" involves a series of derived equivalence relations between each word in the rule and the event to which it refers, with specific words in the rule such as when and then functioning as contextual cues for temporal relations. In this case, the rule specifies "green light first, go second."

In apparently resolving the conceptual question of how rules specify contingencies, RFT pushed the more basic concept of derived relations center stage in terms of dealing with human psychological suffering. Early ACT, therefore, started with a very strong focus on rule-governed behavior and the problems it created for psychological suffering (e.g., resulting in the view that suffering involved excessive rule following), but in grappling with the concept of rule-governed behavior itself, the role of derived relations in human suffering became increasingly apparent. Perhaps the clearest example of this was the concept of the transformation of functions that is central to RFT.

\section{Transformations of Functions}

One area in which the transformation of functions has often been invoked in the clinical domain is in understanding the development and maintenance of irrational fears and 
phobias (e.g., Augustson \& Dougher, 1997; Dougher, Augustson, Markham, Greenway, \& Wulfert, 1994). To illustrate, let us consider a simple example of an early, relatively minor traumatic event. Imagine a boy who went horse riding for the first time, experienced a bad fall from the horse, and subsequently started to fear horses. In this case, the fear of horses could be considered to be directly conditioned. Now imagine that the boy spontaneously develops a fear of riding his bicycle, even though he has not experienced a fall while doing so. Such a transfer of aversive or fear functions could be based, at least in part, on the fact that horses and bicycles participate in a frame of equivalence or coordination in the context of "things you ride." Indeed, in time, it is possible that the boy may avoid learning to ride a motorcycle or perhaps even drive a car because these all enter into an equivalence frame of "modes of transport" that may lead to injury.

Indeed, the transformation of functions can also be used to explain increasingly complex examples of psychological suffering. For instance, the ability to relate entire relational networks to other relational networks may be involved in highly abstract transformations of functions. To illustrate, imagine a woman who has recently begun to feel trapped in a number of areas of her life (e.g., work, relationships, and family responsibilities). The use of the word trapped in these contexts of her life results in bouts of claustrophobia and panic when she is required to enter enclosed spaces, including elevators, subways, and crowded shopping areas. The emergence of claustrophobia and panic may have little to do with actual aversive experiences in any of these contexts but is based on the transformation of functions of those contexts because they are coordinated through the term trapped to the relational networks that describe work, relationships, and family responsibilities.

When human psychological suffering is interpreted or explained in terms of derived relations and the derived transformation of functions, it highlights the importance of focusing on the role of language during the process of therapy. In the case of the woman who has developed claustrophobia and panic described previously, in the context of feeling trapped in several domains of her life, it may be useful in therapy to explore the word trapped itself. For example, in exploring the functional properties of trapped, the therapist might literally hold the client's wrists gently and ask her to describe how it feels to be trapped by someone else. Engaging in this physical metaphor may help the client to see the connections between her claustrophobia or panic and the wider unhappy features of her life and to then explore her reactions to these in ways that in terms of ACT are defined as values driven rather than values disabling (e.g., consider changing jobs, sharing her fears with her partner, and so on).

\section{Rule-Governed Behavior}

In recognizing that the concept of the transformation of functions may help to explain certain features of human psychological suffering, the role of rules in such suffering was seen in a fresh light. Of course, we learn to follow rules from an early age, and this provides us with useful strategies for controlling our own behavior and predicting the behavior of others. For example, parents pass on rules to their children about poisonous foods such as raw meats (e.g., if chicken is undercooked, it can make you sick) so they can avoid serious illness without making direct contact with the natural contingencies. 
According to the ACT literature, rule-governed behavior may also create behaviors that are insensitive to contingencies, such that it helps to promote psychological suffering rather than protect us from potentially harmful events in the natural environment. Indeed, many researchers have proposed that persistent rule following and the insensitivity effect may help to explain specific clinical behaviors (McAuliffe, Hughes, \& Barnes-Holmes, 2014, p. 2). According to ACT, therefore, excessive reliance on multiple rules in one's day-to-day life may become problematic. Consider, for example, a man who attempts to adhere to the following types of rules:

- "I must always be strong."

- "I can't let my guard down."

- "People can never see me upset."

- "Men shouldn't get upset."

This type of rule following may indeed work well in his professional life because he is the leader of a team. However, in a different context of his life, such as his relationship with his partner, following these rules excessively becomes problematic because he never talks about things that upset him or appear to make him vulnerable, which in turn influences his level of intimacy with his partner.

In the context of therapy, ACT uses various interventions to undermine excessive rule following. The therapist does this by helping the client to identify exemplars of rule following and by exploring, in ACT terms, the workability of the rule in various contexts (i.e., What are the consequences of the rule following?). For example, using the previous example, the therapist identifies the rule "People can never see me upset" as controlling the client's behavior. To identify the extent of the control of the rule, the therapist might ask questions such as "Can you give me some examples of when you might use this rule?" The therapist can then begin to target the behavioral control functions of the rule by asking questions such as "Is it possible that living according to this rule might have actually pushed your partner away from you?" These questions can offer the client an alternative - or in terms of ACT - a defused perspective from which to view his own behavior in the context of the rule. The therapist might then try to encourage the client to engage in alternative contingency-sensitive behaviors (rather than rule-consistent behaviors), such as shaping up the behavior of talking to his partner about his feelings through successive approximations. For example, the client might first be encouraged to share with his partner when he had a bad day, and over time, he might be encouraged to be able to cry in front of his partner if he feels the need to.

\section{Relating Relations and Analogy}

The previous section highlights how rules, as networks of derived stimulus relations, have been used to understand and treat human psychological suffering within ACT. Another area in which the study of derived relations has been closely tied to ACT is in developing an RFT account of analogical reasoning and metaphor. According to RFT, relating derived relations lies at the root of analogies and metaphors (Stewart \& BarnesHolmes, 2001). To illustrate this basic account, let us consider the simple analogy of "peach is to pear as cat is to dog," in which one coordination relation is related to 
another coordination relation. One coordination relation relates two stimuli in the context of fruit, and the other coordination relation relates the stimuli in the context of domestic animals. The phrase is to relates the two relations as coordinate with each other. Critically, the four stimuli do not "collapse" into a single relational network in which all four elements become equivalent or coordinate - instead, the network consists of two separate relations that are related to each other as relations.

One of the key functions of analogy and metaphor in natural language is to help listeners to use established knowledge in one domain to help understand an event or information in another domain. The classic Rutherfordian analogy in which the structure of atoms was likened to planets orbiting the sun provides one example in the context of science. Relating relations, as the basis of analogy and metaphor, has also been used to help clients to see their psychological suffering in new or different ways that may facilitate clinical change (see Foody et al., 2014). To illustrate, let us consider one of ACT's stock analogies: "Struggling with anxiety is like struggling in quicksand." This example contains the relevant elements: (a) two coordination ${ }^{2}$ relations (struggling with anxiety or panic and struggling in quicksand drowning), (b) a coordination relation between these relations (struggling with anxiety is like struggling in quicksand), and (c) seeing "struggling" as part of the problem. More technically, the analogy is designed so that the functions of struggling in quicksand transfer to struggling with anxiety through the coordination-coordination relation. Put simply, the analogy suggests that there is something about struggling with anxiety that from the therapist's perspective is similar to struggling in quicksand. Specifically, the urge to struggle to escape quicksand typically leads to more rapid drowning, just as struggling with anxiety may lead to the rapid onset of a full-blown panic attack. This type of analogy could be useful in therapy if a client had not previously seen that attempts to struggle to control anxiety might actually increase the likelihood of descending into panic. Asking the client to consider the analogy or to relate stimulus relations in this way may thus encourage the client to respond differently to the experience of anxiety when it occurs. Relating relations, in the context of this analogy, thus suggests that it may be useful to expose oneself to anxiety in much the same way as one survives in quicksand by lying relatively still and not struggling.

An important caveat to the use of relating relations in therapy is that it occurs in the wider context of the therapist conducting a functional assessment of the client's situation. In other words, the therapist needs to identify the client's key problem. For example, if the client's anxiety does not include a sense of struggling to breathe, then the coordination with drowning in quicksand will likely not work. Similarly, if a client has never heard of quicksand, then there is no point in trying to construct a relational network around this. Critically, the more closely the analogy matches the relevant relational networks for the client, the more likely it is that the appropriate behavioral change (in this case, transformation of functions for the word struggle) will occur for the client.

\footnotetext{
${ }^{2}$ For the purposes of this example, these are described as coordination relations, which are simple relational networks, and once these networks are expanded within the context of therapy, the networks become more complex. Thus, I will use the terms relations and networks almost interchangeably.
} 


\section{Deictic Stimulus Relations and the Verbal Self}

Skinner (1974) first referred to self-awareness as produced by social contingencies that reinforce the discrimination of one's own behavior, out of which the sense of self becomes abstracted (see also Dymond \& Barnes, 1997). The emergence of a stable sense of self is a critical feature of human development and an assumed prerequisite for complex verbal behavior and psychological well-being (Dymond \& Barnes, 1997; Hayes, 1984). And clinical researchers have often argued that a fractured or deviant development of the self can be associated with psychological suffering (e.g., Ingram, 1990; McEnteggart, Barnes-Holmes, Dillon, Egger, \& Oliver, 2017). For RFT, a verbal self involves three functionally distinct deictic relational units: the interpersonal I-you relations, the spatial here-there relations, and the temporal now-then relations (BarnesHolmes, 2001). The basic idea is that the verbal community gradually teaches a young child to distinguish himself or herself from others and to locate this verbal self in both space and time. For example, young children are frequently asked by caregivers:

- "What are you doing now?"

- "What did you do then?"

- "Where are you going tomorrow?"

- "Did you go there with your dad or your mom?"

As a child learns to respond appropriately to these questions, the verbal self, located in time and place and in relation to others, emerges in the behavioral repertoire.

It has been argued that the verbal self (sometimes referred to as the "deictic I") appears to be central to psychological suffering (e.g., Barnes-Holmes et al., 2018). From an RFT perspective, this suffering may emerge when the verbal self participates in certain instances of excessive rule following. For example, the rule or relational network "Only bad people end up alone" may help to create a negative evaluation of the verbal self following a divorce or the death of a spouse. More technically, the excessive rule following produces a transformation of negative evaluative functions of the self based on coordinating the verbal self with alone and alone with badness. In ACT, this effect may be referred to as fusion with negative thoughts and feelings about the self. Critically, these negative evaluations of the self, as instances of excessive rule following, by definition, reduce the likelihood that behaviors will be emitted that could bring the individual into contact with reinforcement contingencies that would undermine the problematic rule following. For example, the individual engaging in new social activities following a divorce may be less likely if the individual believes that he or she deserves to be alone because he or she is a bad person and not worth loving.

One way in which ACT tackles this type of fusion or excessive rule following is to help the client appreciate that verbal rules are simply rules and that, in a sense, the verbal self is free to choose whether or not to follow them. The aim here is not to challenge the problematic rule itself but to highlight that the verbal self is separate or distinct from any rule or relational network in which the self may participate. Indeed, in ACT, certain metaphors or analogies are sometimes used to make this point. For example, one common metaphor involves suggesting that the verbal self is like a chessboard upon which the black and white pieces represent negative and positive rules or evaluations about the self. In this metaphor, the board provides a context for the 
rules but is separate from them (the chessboard metaphor is sometimes referred to as a self-as-context exercise in the ACT literature). The aim here is to undermine excessive rule following that may limit or reduce a client's contact with potential reinforcers in the natural environment.

\section{The Relationship Between the Verbal Self and Others}

The development of the relationship between the verbal self and others also appears to be critical in the emergence of certain instances of psychological suffering (BarnesHolmes et al., 2018; McEnteggart et al., 2017). Imagine a young boy who is subject to physical and emotional abuse by a parent over a period of years. The parent would often abuse the child in one moment and then might say "You know that I love you" the next moment. The fact that the parent emits relational networks or rules pertaining to the child's verbal self (i.e., that he is loved) in a way that is incoherent with how the wider verbal community responds to such networks (most people do not routinely abuse people they love) may undermine the child's ability to connect in a healthy way with others in adulthood. That is, the child may find it challenging in later life to form a close and intimate relationship with someone who is not abusive toward him in some way. In extreme cases, the levels of relational incoherence that are created by highly abusive parenting may alter the development of a coherent or stable verbal self, resulting in severe psychological manifestations such as hearing voices, dissociation, or paranoia (McEnteggart et al., 2017).

When confronted with such extreme cases of psychological trauma, an ACT therapist may focus extensively on the therapeutic relationship as a means of attempting to establish a more coherent verbal self for the client. This is typically achieved by creating an intensely cooperative environment in which the therapist provides a verbal environment devoid of the incoherent relational networks that characterized the client's early family experiences. In a sense, the therapist aims to construct a relationally coherent and stable verbal sense of self for the client that failed to emerge due to abusive parenting. This can be extremely challenging work for a therapist (and indeed the client) because it may involve contacting relatively aversive functions for both individuals during the course of therapy. For example, a therapist might say, "I can almost feel how painful that would have been for you; if I were in your shoes, I would find it very difficult to trust that someone really loves me now." Indeed, this sharing of potentially aversive relational networks pertaining to the impact of an abusive history is likely critical for successful therapeutic outcomes in such extreme cases.

\section{A New Framework in RFT and Its Implications for ACT}

This section will briefly consider a new framework within RFT that emphasizes the potentially dynamic nature of derived relational responding. In my view, this new development may help to reconnect RFT more directly with ACT, specifically the types of clinical phenomena that arise in therapy. The framework is referred to as the multidimensional multilevel (MDML) framework (see Barnes-Holmes et al., in press, in the current special issue for an illustrated figure of the framework). According to the MDML 
framework, there are five levels of relational responding: (a) mutual entailing, (b) relational framing (simplest relational network), (c) relational networking, (d) relating relations, and (e) relating relational networks. The framework also conceptualizes each of these levels as having four dimensions: (a) derivation, (b) complexity, (c) coherence, and (d) flexibility. Each level intersects with each dimension, yielding 20 units of analysis for conceptualizing the dynamics of relational responding (see Barnes-Holmes, Barnes-Holmes, Luciano, \& McEnteggart, 2017, for a detailed treatment of the MDML framework). In brief, derivation refers to how many times a derived response has been emitted - the first response is, by definition, high in derivation because it derived entirely from a trained relation(s). Subsequently, however, derived responses gradually acquire their own history and thus are less and less derived from the initially trained relation(s). Complexity refers to the detail or density of a pattern of relational responding, such as the number of relations and/or different types of relations in a given network. Coherence refers to the extent to which relational responding is generally predictable or consistent with previously established patterns of relational responding (whether directly trained and/or derived). Flexibility refers to the extent to which patterns of derived relational responding may be altered or affected by various contextual variables (e.g., how readily a pattern of equivalence responding may change when the trained baseline relations are reversed).

To appreciate how the MDML framework serves to emphasize the dynamics that may emerge in studying derived relational responding in basic experimental research, the reader should consult the study by Barnes-Holmes et al. (2017). For present purposes, however, I will focus on how the MDML framework connects directly to clinically relevant phenomena. Imagine a client who comes into therapy and in the first session says, "I am a terrible person." The therapist replies with "Do you really believe this?" and the client answers, "Without a doubt, I truly am bad to the bone." The therapist then asks, "How long have you thought this?" and the client replies, "As long as I can remember." The therapist then asks, "Why do you think you are so bad?" and the client replies, "I don't know really, I just am." Finally, the therapist says, "That's funny you say that because you don't seem like a bad person to me," to which the client reacts slightly aggressively and says, "But you don't even know me yet; when you do, you'll see how bad I really am."

How might we conceptualize this therapeutic exchange in terms of the MDML framework? First, when the client says, "I am a terrible person," this may be defined as mutually entailing the verbal self with terrible. Second, when the client states "Without a doubt, I truly am bad to the bone," the mutual entailing may be defined as high in coherence (i.e., it is highly consistent with other things that the client says about himself). Third, when the client indicates that he has been thinking this for "As long as I can remember," the mutually entailing is defined as low in derivation (i.e., the client has been thinking this almost habitually). Fourth, when the client says, "I just am" when asked why he thinks he is bad, the mutual entailing is defined as relatively simple (low in complexity) at that point in the therapeutic exchange. Finally, when the client reacts negatively to the therapist suggesting that he does not seem like a bad person, the mutual entailing may be defined as highly inflexible.

The MDML may be used to conceptualize relatively subtle differences in the aforementioned type of therapeutic exchange. Imagine, for example, that when the client was asked why he says he is a bad person that he provided a long list of reasons (rather than simply saying "I just am"). The response might be better 
categorized as relational networking or even relating relational networks if the client said, 'I'm divorced, my kids don't talk to me, I drink too much, I don't work hard and think I might get fired, and when my mom died last year I was too drunk to attend the funeral". In terms of dimensions, the client's responding might be defined as low in coherence if he responded differently to the therapist's question "Do you really believe this?" and said "I mean, I think I am; I do try to be a better person though." Second, if the client indicated that it had only recently dawned on him that he was a bad person (rather than thinking this for years), the network might be considered relatively high in derivation (i.e., as a verbal response that had only emerged recently in the client's verbal repertoire). Third, because the client replied with a long list of reasons of why he is bad, the networking may be defined as high in complexity. And if the therapist challenges the client on this and the client instead replies with "Maybe you're right; my sister says the same thing to me," this may indicate a higher level of flexibility than that presented in the previous example.

At the time of writing, the MDML framework was relatively new in the literature on derived relational responding, and thus it remains to be seen how much it affects ACT as practiced in a clinical setting. Nevertheless, it seemed useful to present this recent development in this article because it illustrates that there are ongoing efforts to connect the study of derived stimulus relations with ACT and with therapy in general.

\section{Conclusions}

The primary aim of this article was to present a brief tutorial on ACT as seen through the lens of derived stimulus relations and RFT in particular. As noted at the beginning of the article, the relationship between ACT and RFT appeared to be relatively direct in the early years of both research programs, but there was an undeniable drift between the two, which made it increasingly difficult to see how ACT was rooted firmly in the basic science concepts surrounding the study of derived stimulus relations. Nevertheless, connections do remain, and it is possible that the MDML framework and other conceptual developments in RFT may help to further strengthen and clarify these connections. Focusing on these connections will be important for the basic science in terms of maintaining its pragmatic utility and equally important for the application in terms of ensuring that it continues to progress with developments in the basic science.

\section{References}

Augustson, E. M., \& Dougher, M. J. (1997). The transfer of avoidance evoking functions through stimulus equivalence classes. Journal of Behavior Therapy and Experimental Psychiatry, 28, 181-191.

Baer, D. M., Wolf, M. M., \& Risley, T. R. (1968). Some current dimensions of applied behavior analysis. Journal of Applied Behavior Analysis, 1, 91-97.

Barnes-Holmes, D., Barnes-Holmes, Y., Luciano, C., \& McEnteggart, C. (2017). From the IRAP and REC model to a multi-dimensional multi-level framework for analyzing the dynamics of arbitrarily applicable relational responding [special issue]. Journal of Contextual Behavioral Science, 6, 434-445. 
Barnes-Holmes, D., Finn, M., McEnteggart, C., \& Barnes-Holmes, Y. (in press). Derived stimulus relations and their role in a behavior-analytic account of human language and cognition [special issue]. Perspectives on Behavioral Science.

Barnes-Holmes, Y. (2001). Analysing relational frames: studying language and cognition in young children (unpublished doctoral thesis). National University of Ireland, Maynooth.

Barnes-Holmes, Y., Boorman, J., Oliver, J., Thompson, M., McEnteggart, C., \& Coulter, C. (2018). Using conceptual developments in RFT to direct case formulation and clinical intervention: two case summaries [special issue]. Journal of Contextual Behavioral Science, 7, 89-96.

Barnes-Holmes, Y., Hussey, I., McEnteggart, C., Barnes-Holmes, D., \& Foody, M. (2016). Scientific ambition: the relationship between relational frame theory and middle-level terms in acceptance and commitment therapy. In R. D. Zettle, S. C. Hayes, D. Barnes-Holmes, \& A. Biglan (Eds.), The Wiley handbook of contextual behavioral science (pp. 365-382). West Sussex: Wiley-Blackwell.

Beck, A. T. (1976). Cognitive therapy and the emotional disorders. New York, NY: International Universities Press.

Bond, F. W., Hayes, S. C., \& Barnes-Holmes, D. (2006). Psychological flexibility, ACT, and organizational behavior. Journal of Organizational Behavior Management, 26(1-2), 25-54.

Burke, K., Muscara, F., McCarthy, M., Dimovski, A., Hearps, S., Anderson, V., \& Walser, R. (2014). Adapting acceptance and commitment therapy for parents of children with life-threatening illness: pilot study. Families, Systems, \& Health, 32, 122-127.

Carr, E. G. (1977). The motivation of self-injurious behavior: a review of some hypotheses. Psychological Bulletin, 84, 800-816.

Carr, E. G. (1994). Emerging themes in the functional analysis of problem behavior. Journal of Applied Behavior Analysis, 27, 393-399.

Dougher, M. J., Augustson, E., Markham, M. R., Greenway, D. E., \& Wulfert, E. (1994). The transfer of respondent eliciting and extinction functions through stimulus equivalence classes. Journal of the Experimental Analysis of Behavior, 62, 331-351.

Dymond, S., \& Alonso-Álvarez, B. (2010). The selective impact of Skinner's Verbal Behavior on empirical research: a reply to Schlinger (2008). The Psychological Record, 60, 355-360.

Dymond, S., \& Barnes, D. (1997). Behavior-analytic approaches to self-awareness. The Psychological Record, 47, 181-200.

Dymond, S., O'Hora, D., Whelan, R., \& O’Donovan, A. (2006). Citation analysis of Skinner's Verbal Behavior: 1984-2004. The Behavior Analyst, 29, 75-88.

Feros, D. L., Lane, L., Ciarrochi, J., \& Blackledge, J. T. (2013). Acceptance and commitment therapy (ACT) for improving the lives of cancer patients: a preliminary study. Psycho-Oncology, 22, 459-464.

Foody, M., Barnes-Holmes, Y., Barnes-Holmes, D., Törneke, N., Luciano, C., Stewart, I., \& McEnteggart, C. (2014). RFT for clinical use: the example of metaphor. Journal of Contextual Behavioral Science, 3, 305313.

Friman, P. C. (2006). The future of behavior analysis is under the dome. Association for Behavior Analysis International Newsletter, 29, 4-5.

Hanley, G. P., Iwata, B. A., \& McCord, B. E. (2003). Functional analysis of problem behavior: a review. Journal of Applied Behavior Analysis, 36, 147-185.

Hayes, S. C. (1984). Making sense of spirituality. Behavior, 12, 99-110.

Hayes, S. C. (Ed.). (1989). Rule-governed behavior: cognition, contingencies, and instructional control. New York, NY: Guilford Press.

Hayes, S. C. (2001). The greatest dangers facing behavior analysis today. The Behavior Analyst Today, 2, 6163.

Hayes, S. C., Barnes-Holmes, D., \& Roche, B. (2001). Relational frame theory: a post-Skinnerian account of human language and cognition. New York, NY: Kluwer Academic.

Hayes, S. C., Luoma, J. B., Bond, F. W., Masuda, A., \& Lillis, J. (2006). Acceptance and commitment therapy: model, processes and outcomes. Behaviour Research and Therapy, 44, 1-25.

Hayes, S. C., Rincover, A., \& Solnick, J. V. (1980). The technical drift of applied behavior analysis. Journal of Applied Behavior Analysis, 13, 275-285.

Hayes, S. C., Strosahl, K., \& Wilson, K. G. (1999). Acceptance and commitment therapy: understanding and treating human suffering. New York, NY: Guilford Press.

Hughes, S., \& Barnes-Holmes, D. (2016). Relational frame theory: the basic account. In R. D. Zettle, S. C. Hayes, D. Barnes-Holmes, \& A. Biglan (Eds.), The Wiley handbook of contextual behavioral science (pp. 129-178). West Sussex: Wiley-Blackwell.

Ingram, R. E. (1990). Self-focused attention in clinical disorders: review and a conceptual model. Psychological Bulletin, 107, 156-176. 
Iwata, B. A., Dorsey, M. F., Slifer, K. J., Bauman, K. E., \& Richman, G. S. (1982). Toward a functional analysis of self-injury. Analysis and Intervention in Developmental Disabilities, 2, 3-20.

Lillis, J., \& Hayes, S. C. (2007). Applying acceptance, mindfulness, and values to the reduction of prejudice: a pilot study. Behavior Modification, 31, 389-411.

Luiselli, J. K., \& Cameron, M. J. (1998). Antecedent control: innovative approaches to behavioral support. Baltimore, MD: Brookes.

Lundgren, T., Dahl, J., \& Hayes, S. C. (2008). Evaluation of mediators of change in the treatment of epilepsy with acceptance and commitment therapy. Journal of Behavioral Medicine, 31, 225-235.

McAuliffe, D., Hughes, S., \& Barnes-Holmes, D. (2014). The dark-side of rule governed behavior: an experimental analysis of problematic rule-following in an adolescent population with depressive symptomatology. Behavior Modification, 38, 587-613.

McEnteggart, C., Barnes-Holmes, Y., Dillon, J., Egger, J., \& Oliver, J. E. (2017). Hearing voices, dissociation, and the self: a functional-analytic perspective. Journal of Trauma \& Dissociation, 18, 575-594.

O’Connor, M., Farrell, L., Munnelly, A., \& McHugh, L. (2017). Citation analysis of relational frame theory: 2009-2016. Journal of Contextual Behavioral Science, 6, 152-158.

O’Neill, R. E., Horner, R. H., Albin, R. W., Story, K., \& Sprague, J. R. (1990). Functional analysis of problem behavior: a practical assessment guide. Sycamore, IL: Sycamore Publishing.

Sidman, M. (1971). Reading and auditory-visual equivalences. Journal of Speech, Language, and Hearing Research, 14, 5-13.

Sidman, M. (1994). Equivalence relations and behavior: a research story. Boston, MA: Authors Cooperative.

Skinner, B. F. (1966). The phylogeny and ontogeny of behavior. Science, 153, 1205-1213.

Skinner, B. F. (1969). Contingencies of reinforcement: a theoretical analysis. New York, NY: AppletonCentury-Crofts.

Skinner, B. F. (1974). Walden two. Indianapolis, IN: Hackett Publishing.

Stewart, I., \& Barnes-Holmes, D. (2001). Understanding metaphor: a relational frame perspective. The Behavior Analyst, 24, 191-199.

Törneke, N. (2010). Learning RFT: an introduction to relational frame theory and its clinical application. Oakland, CA: New Harbinger.

Villatte, M., Villatte, J. L., \& Hayes, S. C. (2015). Mastering the clinical conversation: language as intervention. New York, NY: Guilford Press.

Zettle, R. (2016). Contextual approaches to clinical interventions and assessment: an introduction to part III. In R. D. Zettle, S. C. Hayes, D. Barnes-Holmes, \& A. Biglan (Eds.), The Wiley handbook of contextual behavioral science (pp. 275-286). West Sussex: Wiley-Blackwell.

Zettle, R. D. (1990). Rule-governed behavior: a radical behavioral answer to the cognitive challenge. The Psychological Record, 40, 41-49.

Zettle, R. D., \& Hayes, S. C. (1983). Effect of social context on the impact of coping self-statements. Psychological Reports, 52, 391-401. 